\title{
MONET: a MOnitoring NEtwork of Telescopes
}

\author{
Frederic V. Hessman \\ Universitäts-Sternwarte, Geismarlandstr. 11, 37083 Göttingen, Germany
}

\begin{abstract}
In an age of $8 \mathrm{~m}$-class telescopes, we should rethink the way we use small telescopes. While $2-4 \mathrm{~m}$ telescopes are still needed and will continue to be operated largely in the traditional fashion, new $1 \mathrm{~m}$-class telescopes operated robotically in global networks will enable a wide range of new and exciting scientific and educational projects, both by themselves and in conjunction with much larger telescopes. I describe our plans for such a global network of two $1 \mathrm{~m}$-class robotic telescopes.
\end{abstract}

\section{Introduction}

The research tools of an astrophysicist at the beginning of the 21st century are very different from those of his/her colleague a decade or two earlier. In Göttingen, we now routinely use the 8m ESO Very Large Telescopes in Chile and the $9.2 \mathrm{~m} \mathrm{Hobby-Eberly-Telescope} \mathrm{in} \mathrm{Texas} \mathrm{and} \mathrm{will} \mathrm{soon} \mathrm{have} \mathrm{access} \mathrm{to} \mathrm{the}$ Southern African Large Telescope and the Large Binocular Telescope in Arizona. A wide range of large-aperture ground and space telescopes covering almost the entire range of wavelengths is available or on the horizon. The present $2-4 \mathrm{~m}$-class ("medium-sized") telescopes are still needed, both for their own sakes and for supporting observations for the larger telescopes. And, of course, many institutes still have working $0.5-1.5 \mathrm{~m}$ (a modern definition of "small") telescopes.

The dramatic increase in the available telescope area per working astronomer has not been accompanied by an equal increase in our ability to carry out more projects or sub-projects simultaneously. Even though computers have made it possible to process Terrabytes of data almost routinely, our mental capacity to plan, organize and carry out observations is limited (a simple "zero-sum" mental model of an astrophysicist). Thus, given the finite number of things we can do in a typical working day, is there any place for general-purpose small telescopes in an age of $8 \mathrm{~m}$ giants?

Most of the participants at this colloquium wouldn't be here if they thought the answer was "no", but it is instructive to consider what service small telescopes can and should still provide and what constraints on their operation are now relevant.

\section{Support observations for $4-10 \mathrm{~m}$ telescopes:}

if such observations are not to be a burden on the preparations for and analysis of larger-telescope observations, they must be easy to obtain. Since the large telescopes are based on both hemispheres, simple and effective access to telescopes on both hemispheres becomes very important. 


\section{Projects which require large numbers of observations:}

since our access to $4-10 \mathrm{~m}$ telescopes is limited, this is a natural field for small telescopes, but such observations must be easy to manage and have low personnel/travel costs. A classic example of such a project and the inherent costs involved with performing them using traditional observational methods is the COYOTES collaboration (e.g. Bouvier et al. 1997): while their monitoring of the photometric modulations of pre-main sequence stars defined our knowledge of the rotational evolution of such stars, that knowledge came at a considerable personnel and financial cost.

\section{Projects which require all-sky or extended time coverage:}

large-scale surveys, simultaneous observations with spacecraft, and observations of phenomena on timescales greater than a night require access to the whole sky. (the WET Consortium is a good example of the need for both sky and time coverage: see the article by Kawaller). The organization of loose consortia for this type of work (e.g. AGN monitoring) has traditionally been painful and often unsuccessful. One needs automatic access to several telescopes spread out in latitude and longitude.

\section{Projects which require extreme flexibility:}

an immediate access to the telescope is required, e.g. to detect the optical counterparts of $\gamma$-ray bursts or to follow the lightcurve of a supernova or a micro-lensing event. Immediate access is usually only possible when one has guaranteed access - usually only available on small telescopes.

\section{Limited access to larger telescopes:}

ideally, this should not be a major constraint. In practice, our colleagues from less-developed countries have a difficult time finding travel funds. As more telescopes become remotely and/or robotically operated, the opportunities to do interesting science will hopefully increase significantly. Thus, we can hope that this reason to use small telescopes will diminish with time.

\section{Training:}

the best way to produce future generations of instrument builders is to let undergraduate and graduate students have direct access to working telescopes and the possibility to construct and manipulate low-cost but relatively "state-of-the-art" instrumentation.

\section{Education:}

even when cutting-edge science is mostly done with larger telescopes, small telescopes can be used very effectively by high school and even elementary school students in programs which enable them to learn about the scientific process "hands-on".

While Training requires direct access to telescope hardware, all of the other small telescope uses do not or indeed are only possible when several telescopes distributed over the globe are at least remotely steerable and even fully robotic and autonomous. Thus, the future of small telescope astronomy lies in the use of global networks of robotic telescopes. 


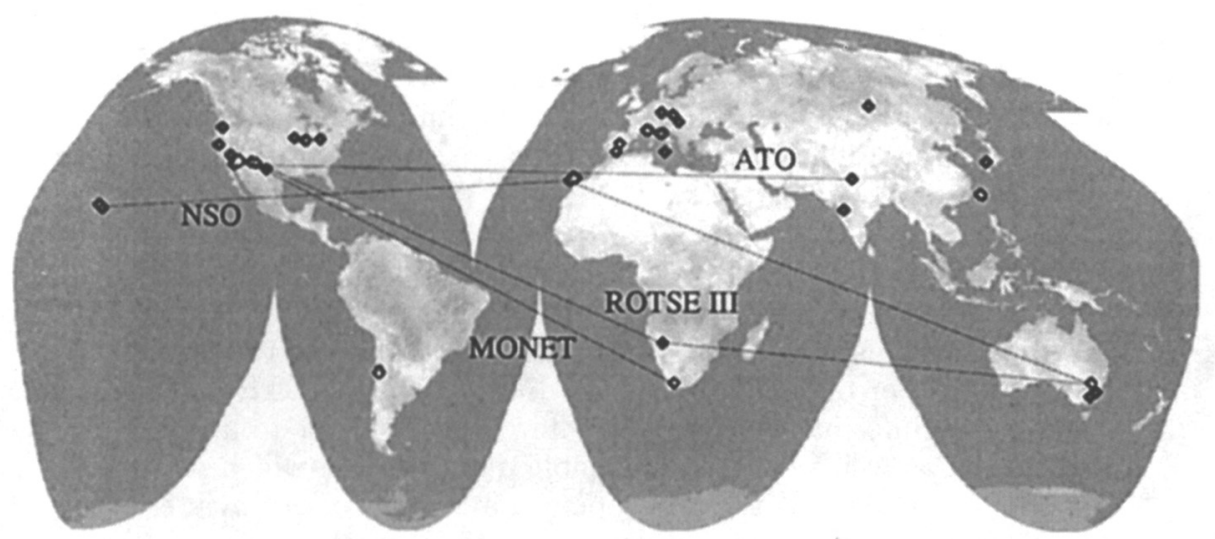

Figure 1. A map showing the global distribution of robotic telescopes. The networks are indicated by the connected sites: MONET is our own project; ROTSE III is the planned extension of the ROTSE experiment, ATO is the Antipodal Telescope Observatory, and NSO is the National Schools Observatory (see the table of robotic telescopes in the appendix).

\section{Robotic Telescopes}

While there might be slight nuances in the definition of what constitutes a "robotic" telescope, the basic definition is that it performs the observations given to it without the need for any local or remote operator. This means that the system must respond (quickly) to changes in weather and must gather it's own calibration observations. Modern robotic telescopes should be able to handle their own scheduling dynamically - e.g. to respond to a Targetof-Opportunity $(\mathrm{ToO})$ request without losing track of what it should be doing otherwise or to respond to changing seeing and weather conditions and not just blindly go through a list of proposed targets. Robotic telescope systems should perform some level of data-pipelining (dark, bias, flatfield, flux and astrometric calibration) and analysis (photometry of target objects, search for variability or motion across the sky).

There are nearly 60 robotic (or "autonomous") telescopes around the world either in operation, under construction, financed, or planned (see Fig. 1, derived from the table of robotic telescopes in the Appendix) and the number is increasing rapidly. This does not include a number of telescopes which are nearly robotic - e.g. which are capable of performing a long list of observations once a local operator has opened the dome and prepared everything.

The first generations of robotic telescopes - e.g. the Automatic Photometric Telescopes (APT) at Fairborn Observatory developed by Lou Boyd and others (Boyd, Genet \& Hall 1986; Genet, Boyd \& Hall 1986), the Berkeley supernovasearch telescopes (Richmond, Treffers \& Filippenko 1993), the Univ. of Indiana's RoboScope (Honeycutt 1994; Honeycutt et al. 1994ab), and the Iowa Robotic Telescope Facility (Deleo \& Mutel 1992) to mention just a few - were largely constructed from scratch or used existing hardware and required the writing of the full range of telescope, dome, weather-station, and scheduling software needed to 
operate a robotic telescope. The new generation of robotic telescopes is increasingly being purchased "off the shelf", reducing (but certainly not eliminating) the total effort necessary to bring a working system on-line.

There are many difficult issues associated with the operation of an efficient and simple-to-use robotic network:

\section{The Data Avalanche:}

Robotic telescopes are capable of amassing astounding volumes of data which either must be passed on to a well-organized easily accessible databank (via ftp or on some storage medium) and/or quickly processed into higher level information. The data-flow must be kept to a minimum via a standard as well as a user-extensible data-pipeline which permits some analysis of the data at the telescope site and reduces or obviates the need to transport large amounts of data over the internet.

\section{Flexibility:}

in order to perform whole classes of interesting science, the network must be able to respond rapidly to user-defined events, Targets-of-Opportunity, and be useful as a trigger for large telescope observations.

\section{Robotic Software:}

while it is no longer necessary to write all of the software from scratch for each project - practically all telescope venders now offer some form of robotic capability - there is (still) no turnkey system and the scientific constraints on the software are bound to be different for each telescope and network.

\section{Administration \& Maintenance:}

the administration of the network and projects carried out by the network must be simple. A robotic network with heterogeneous scientific and educational users must be able to handle the different users needs and constraints.

\section{Ease of Use:}

the users must have fast and transparent access to the network and have to deal with a minimum of red tape and overhead. Ideally, a creative user should be able to pose a question, submit the observations needed to answer that question, and receive not the raw data but the analysis of the data if not the answer itself - and all within a minimum amount of waiting time.

\section{Communication within the Network and between Networks:}

while it may be simple to organize the transfer of information between the users, administrators and telescope within a simple robotic system, a complex network of users and multiple telescopes and certainly the communication between different networks of different telescopes using different telescope control software requires an adequate means of transfering information about the observations. 
The latter challenge is being met within an expanding fraction of the robotic telescope community via an eXtensible Markup Language (XML) dialect called Robotic Telescope Markup Language ${ }^{1}$ (RTML). Actually, RTML should be called "Remote (rather than Robotic) Telescope Markup Language", since its true function is not to steer robotic telescopes but to permit the seamless transfer of information about an astronomical observation between telescopes and between networks of telescopes. This protocol looks somewhat like the HyperText Markup Language (HTML) used by internet browsers, is self-defining, easily extensible, and is easily parsed both by computers and by humans. Here is an example of a normal RTML request:

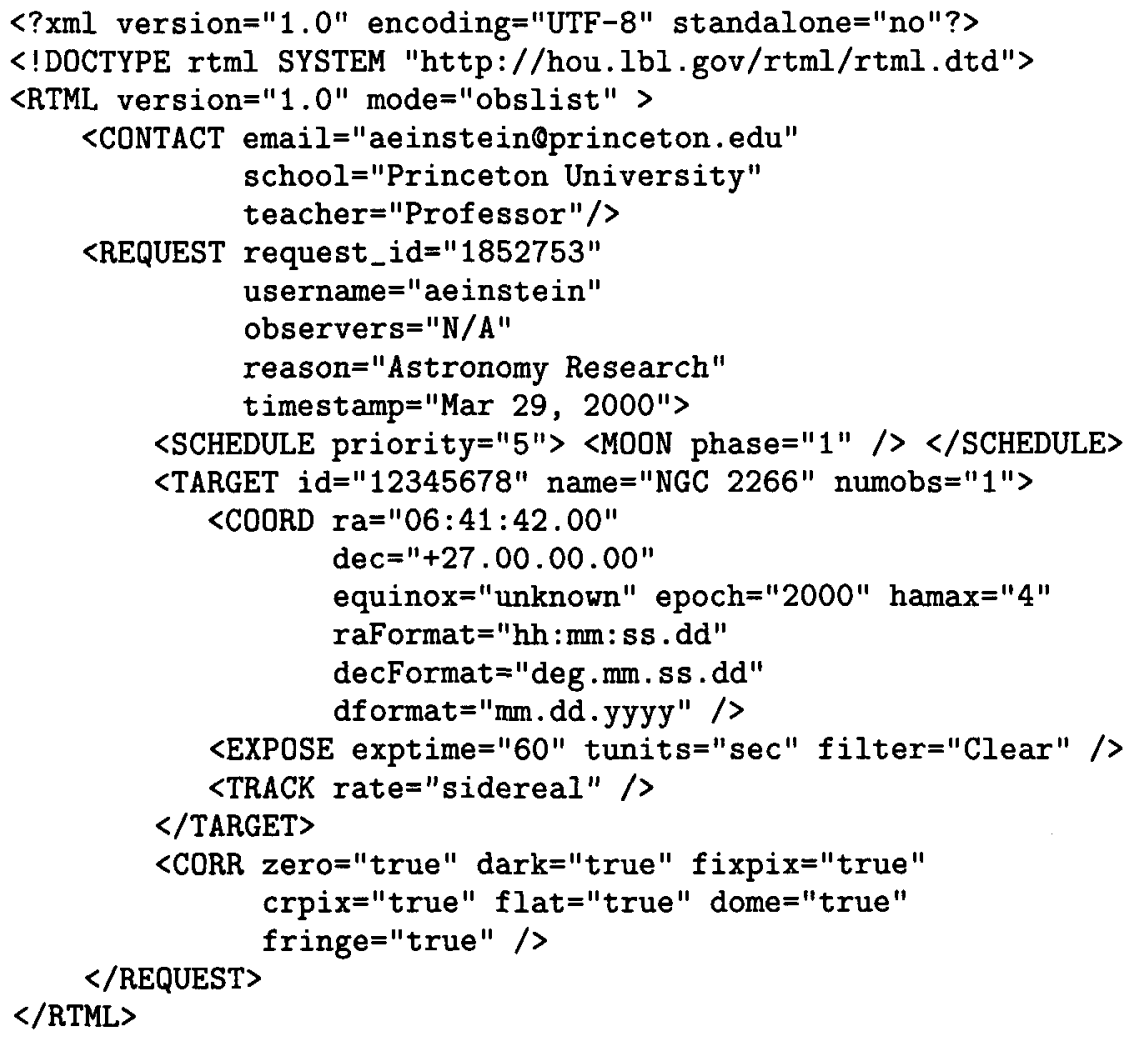

RTML promises to become the universal standard in telescope control and observation specification just as FITS is our standard data format, enabling telescopes and networks using totally different hardware and software to communicate seamlessly.

\footnotetext{
${ }^{1}$ http://hou.lbl.gov/rtml/intro.html
} 


\section{Educational Use of Small Telescopes}

While the participants at the meeting and the readers of the proceedings are undoubtably aware of the many scientific uses of small and particularly robotic telescopes (see, e.g., the many contributions in this volume), they may not be aware of how much activity there has been in the educational use of small telescopes (mostly high school and undergraduate but increasingly even down to elementary school). There are a wide variety of remote and robotic telescopes which are now or will soon be accessible by schools, ranging from quite small "department store"-sized telescopes to $2 \mathrm{~m}$ telescopes.

The most successful remote telescope project is probably "Telescopes In Education" of the Mt. Wilson Observatory. Using standard (commercial) astronomy software for PC's, registered classrooms all over the globe can have access to a 24-inch. While there is no formal curriculum, the project has a well-documented list of interesting projects which can be performed using the data.

The use of remote telescopes in classrooms has the advantage of being immediate and actively usable, but has two substantial problems: (1) the available bandwidth and informal and interactive choice of objects means that the telescope is not used efficiently; and (2) there is the danger of using the telescopes for more astronomical "site-seeing" rather than truly scientific (if small and simple) projects. The attractiveness of astronomy as a natural science with high public awareness and interest should be used to teach how science is done by letting the students do scientific projects. This means placing the goal - answering interesting scientific questions by posing and carrying out a well though-out program of observations and reductions - above the means.

This is the approach taken, e.g. by the Iowa Robotic Telescope Facility ${ }^{2}$ (IRTF) and the Hands-On Universe Project ${ }^{3}$ (HOU). HOU is a privately and federally-funded project complete with a curriculum designed and tested by educators and comes with enough data to carry out astronomical exercises without any telescope at all. HOU is presently expanding its global capacity for providing new data via a global queue system. R. Mutel of the IRTF has written an undergraduate curriculum based on the availability of a robotic queue-driven telescope and is even developing a complete hard- plus software plus curriculum solution (including dome, CCD and spectrograph!) with Torus called Rigel for interested schools, colleges and universities.

\section{The MONET Project}

The reasons why we became interested in robotic telescopes are very simple and probably very typical of many other institutions. While we work in a lovely old observatory and have local access to a old $34 \mathrm{~cm}$ astrograph and a $50 \mathrm{~cm}$ solar telescope, the weather in Göttingen is bad even by German standards. Research groups within the observatory have long needed the monitoring of variable stars

\footnotetext{
${ }^{2}$ http://denali.uiowa.edu

${ }^{3}$ http:///hou.lbl.gov, http://www.uni-sw.gwdg.de/ hou
} 
and AGNs, but the increasing use of $8 \mathrm{~m}$-class telescopes has made it essential to obtain timely and continual information on the photometric state of largetelescope targets. Finally, a drop in the number of incoming physics students has put us under pressure to help improve the quality and hence attractiveness of the physics curriculum in local high schools: we have entered the Hands-On Universe collaboration and have conducted several teacher workshops but can't offer our participating classes any useful access to new data. Thus, we finally decided that we needed our own robotic telescopes for research, university, and school purposes. Since our programs need observations of objects all over the sky, we need at least 2 telescopes stationed on both the northern and southern hemispheres. The observatory already maintains a major solar observatory on Teneriffe, so we are unable to cover the local operating costs of additional telescopes and need partners willing to exchange such support for observing time on both telescopes. Since such partners are primarily interested in the scientific use of the network, the telescopes have to be large enough, e.g., to reach the same faint objects which should be observed spectroscopically with large telescopes.

Given the substantial costs of two telescopes and our intent to use a large fraction of our time for purely educational purposes, Prof. Klaus Beuermann and I approached a private charitable foundation with the idea. The Alfried Krupp von Bohlen und Halbach-Foundation, one of the largest charitable foundations in Germany, generously agreed to be the sole capital contributor - mainly because of the educational benefits promised by the network.

Due to our close contacts with the McDonald and South African Astronomical Observatories via our participation in the construction of the $9.2 \mathrm{~m}$ HobbyEberly-Telescope and with the $9.5 \mathrm{~m}$ Southern African Large Telescope, we have natural northern and southern partners who are very interested in the science which can be done both with the network alone and in conjunction with large telescopes as well as in the educational use.

MONET $^{4}$ will be different from other robotic telescope projects less in the sense that we will do any particular thing differently from any one else but rather because we will combine many of the individual highlights of other projects into a single network:

\section{Sky Coverage:}

we will have complete northern and southern sky coverage with a fully integrated network;

\section{Time Coverage:}

our two sites in the USA and Africa should give us very good longitudinal coverage for extended monitoring programs on timescales longer than a night at a single site;

\section{Direct Connection to 8m-class Telescopes:}

we plan on directly connecting the network to the operation of our two $9 \mathrm{~m}$ telescopes having the same all-sky coverage, HET and SALT, permitting the automatic triggering of large telescope observations via the regular monitoring of our robotic telescopes;

\footnotetext{
${ }^{4}$ www.astro.physik.uni-goettingen.de/ hessman/MONET
} 
Aperture:

we have enough funds for $80-120 \mathrm{~cm}$ telescopes which can easily reach the faint objects which we want to observe spectroscopically with $8 \mathrm{~m}$-class telescopes or provide high $\mathrm{S} / \mathrm{N}$ observations of variables on short timescales - a network of smaller telescopes would make things much more difficult;

\section{International:}

the project will be run as a fully international collaboration;

\section{Education:}

roughly as much time will be given to educational as to scientific projects (a full $50 \%$ of the Göttingen time!); and

\section{Remote \& Robotic:}

our participating schools and our students will have access to the telescopes both remotely and via a robotic queue (with emphasis on the latter, however).

MONET will also help to redefine the use of educational telescope networks: not only will it be available to participating classrooms either remotely or via a queue, we will also provide access to users in external queues like that being set up for the global HOU in order to use up any left-over educational time; we would like to provide a virtual reality access to the telescope in the Göttingen XLAB science center; we plan on targeting young women (the fraction of woman in German physics \& astronomy is one of the lowest in the world); and we want our university students to administer their own fraction of time on the network with their own TACs and projects.

\section{Conclusions}

I have argued that the most effective use of small telescopes in the next decade will come from the operation of networks of robotic, autonomous telescopes. While a decade ago, the construction, programming and operation of such telescopes was a major undertaking made by individual pioneers, the state-of-theart has progressed far enough to make it nearly (but not quite) possible to buy robotic telescopes "off-the-shelf". This permits the creation of homogeneous robotic telescope networks which, thanks to the efforts and good will of many users and venders, will also be connected into very inhomogeneous but powerful networks. The efficient operation and use of these telescopes will make it possible to conduct significant and unique scientific investigations both alone and in combination with large ground- and space-based telescopes. Just as important is the use of the telescopes by schools: we can make it possible for schoolchildren all over the world to learn about science by performing their own and collaborative astrophysical experiments. We hope that MONET - our "MOnitoring NEtwork of Telescopes" which should become operational starting in 2002 - will live up to these high expectations.

I would like to acknowledge the travel support provided by the Deutsche Forschungsgemeinschaft and particularly the generous funding of our project by the Alfried Krupp von Bohlen und Halbach-Foundation. 


\section{References}

Bouvier, J., et al. 1997, A\&A 318, 495

Boyd, L.J, Genet, R.M., Hall, D.S. 1986, PASP 98, 618

Deleo, D.V., Mutel, R.L. 1992, in Robotic Telescopes in the 1990s, ASP Conf. Ser. Vol. 34, e.d. A.V. Filippenko (San Francisco, ASP), 97

Genet, R.M., Boyd, L.J., Hall, D.S. 1986, in Instrumentation and research programmes for small telescopes, Proc. IAU Symp. 118 (Dordrecht, Reidel), 47

Honeycutt, K. 1994, in Optical Astronomy from the Earth and Moon, ASP Conf Series Vol 55, ed. D.M. Pyper, J. Angione (San Francisco, ASP), 103

Honeycutt, R.K., Adams, B.R., Swearingen, D.J., Kopp, W.R. 1994a, PASP, 106,670

Honeycutt, R.K., Robertson, J.W., Turner, G.W., Vesper, D.N. 1994b, in Interacting Binary Stars, ASP Conf Series Vol 56, ed. A.W. Shafter (San Francisco, ASP), 277

Richmond, M, Treffers, R.R., Filippenko, A.V. 1993, PASP 105, 1164

Strassmeier, K.G., Boyd, L.J., Epand, D.H., Granzer, T. 1997, PASP 109, 697 

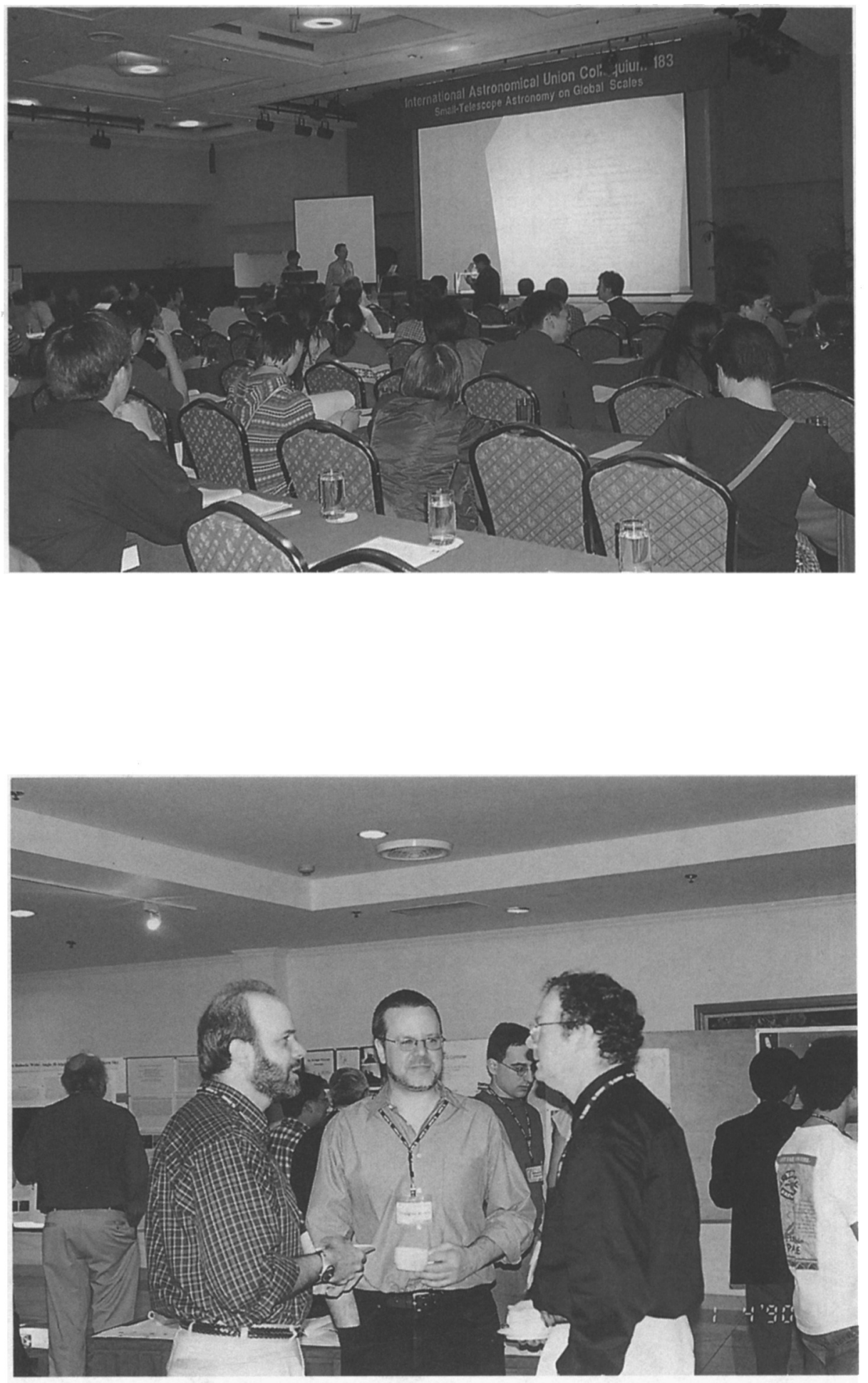

Kawaler, Welch and J. Patterson 